\title{
BMJ Open Defining predatory journals and responding to the threat they pose: a modified Delphi consensus process
}

\author{
Samantha Cukier (10 , ${ }^{1}$ Manoj Lalu (D) , ${ }^{1,2}$ Gregory L Bryson, ${ }^{1,2}$ Kelly D Cobey, ${ }^{1,3}$ \\ Agnes Grudniewicz, ${ }^{4}$ David Moher (i) ${ }^{1,3}$
}

To cite: Cukier S, Lalu M, Bryson GL, et al. Defining predatory journals and responding to the threat they pose: a modified Delphi consensus process. BMJ Open 2020;10:e035561. doi:10.1136/ bmjopen-2019-035561

- Prepublication history and additional material for this paper are available online. To view these files, please visit the journal online (http://dx.doi. org/10.1136/bmjopen-2019035561).

Received 06 November 2019 Revised 20 December 2019 Accepted 16 January 2020

Check for updates

(C) Author(s) (or their employer(s)) 2020. Re-use permitted under CC BY-NC. No commercial re-use. See rights and permissions. Published by BMJ.

${ }^{1}$ Centre for Journalology, Ottawa Hospital Research Institute, Ottawa, Ontario, Canada ${ }^{2}$ Anesthesiology and Pain Medicine, The Ottawa Hospital, Ottawa, Ontario, Canada

${ }^{3}$ School of Epidemiology and Public Health, University of Ottawa Faculty of Medicine, Ottawa, Ontario, Canada

${ }^{4}$ Telfer School of Management, University of Ottawa, Ottawa, Ontario, Canada

Correspondence to

Dr David Moher;

dmoher@ohri.ca

\section{ABSTRACT}

Objective To conduct a Delphi survey informing a consensus definition of predatory journals and publishers. Design This is a modified three-round Delphi survey delivered online for the first two rounds and in-person for the third round. Questions encompassed three themes: (1) predatory journal definition; (2) educational outreach and policy initiatives on predatory publishing; and (3) developing technological solutions to stop submissions to predatory journals and other low-quality journals.

Participants Through snowball and purposive sampling of targeted experts, we identified 45 noted experts in predatory journals and journalology. The international group included funders, academics and representatives of academic institutions, librarians and information scientists, policy makers, journal editors, publishers, researchers involved in studying predatory journals and legitimate journals, and patient partners. In addition, 198 authors of articles discussing predatory journals were invited to participate in round 1.

Results A total of 115 individuals (107 in round 1 and 45 in rounds 2 and 3 ) completed the survey on predatory journals and publishers. We reached consensus on 18 items out of a total of 33 to be included in a consensus definition of predatory journals and publishers. We came to consensus on educational outreach and policy initiatives on which to focus, including the development of a single checklist to detect predatory journals and publishers, and public funding to support research in this general area. We identified technological solutions to address the problem: a 'one-stop-shop' website to consolidate information on the topic and a 'predatory journal research observatory' to identify ongoing research and analysis about predatory journals/publishers.

Conclusions In bringing together an international group of diverse stakeholders, we were able to use a modified Delphi process to inform the development of a definition of predatory journals and publishers. This definition will help institutions, funders and other stakeholders generate practical guidance on avoiding predatory journals and publishers.

\section{INTRODUCTION}

Predatory journals pose a serious threat to legitimate, open access (OA) journals and to the broader scientific community. ${ }^{1}$ They pose as authentic OA journals; however, they often

\section{Strengths and limitations of this study}

An international group of stakeholders participated in a consensus process to define predatory journals and publishers.

- Both empirical evidence and a previous scoping review were used to generate items used in this survey process.

- The last round was held in-person, which allowed for discussion and elaboration of items.

- Although representative of a diverse group of stakeholders, participation in the Delphi was limited.

- Inclusion of a larger number of individuals may have changed the results.

fail to follow usual publication best practices, including peer review and editorial oversight. ${ }^{2}$ These journals have self-interest as a goal, and are often motivated to accept as many articles as possible to profit from article processing charges (APCs) which are common in OA journals. It is becoming increasingly difficult to distinguish articles published in predatory journals from legitimate journals, as predatory journals are also finding their way into trusted sources such as PubMed. ${ }^{3}$

Despite increasing attention to the problem of predatory publishing, ${ }^{4-8}$ there is no agreed-on definition of what constitutes a predatory journal $^{9}$ (however, since the time of publication, a new definition of predatory journals and publishers was established through this Delphi process $\left.{ }^{10}\right)$. The absence of a consensus and operationalised definition makes it difficult to accurately identify and evaluate the problem. Without a definition, funders and academic institutions struggle to generate practical guidance or policy to ensure their members do not publish in predatory journals. Without appropriate attention to the problem of predatory publishing, the quality of scholarly communication is at risk; this includes the risk to researchers, academic institutions and funders, whose credibility 
may be questioned, and/or patients who will have given of their time in hopes of improving interventions or treatments, when in all likelihood these data would not be used. $^{4}$

This paper is part of a programme of scholarship that aims to establish a consensus definition of predatory journals and publishers and establish ways in which the research community should respond to the problem. Cobey and colleagues ${ }^{9}$ reported on the first stage of the programme, which was a scoping review to identify possible characteristics of predatory journals. The authors found that no consensus definition existed and there was a great deal of heterogeneity in the characteristics found. In this study, the second stage of the research programme, we used the characteristics identified from the scoping review to generate a consensus definition of predatory journals and also suggested ways the research community should respond to the problem.

Here we present the details of this modified threeround Delphi consensus study. A related paper describing the consensus statement reached on predatory journals is described elsewhere. ${ }^{10}$

\section{METHODS}

Prior to commencing this study, a protocol was drafted and was posted on the Open Science Framework (https:// osf.io/z6v7f/).

The Delphi method is a structured method to elicit opinions on given questions from a group of experts and stakeholders. ${ }^{11}{ }^{12}$ It is especially useful when exact knowledge is not available. The participants respond anonymously to questionnaires that sequentially incorporate feedback and are refined. Following each round, average group responses are provided to each respondent, allowing them to reconsider their own views on the topic. This is generally performed through electronic survey; however, for our modified Delphi, the final round was held through a face-to-face meeting.

\section{Delphi survey questions: predatory journals and publishers}

The Delphi survey was made up of 18 questions and 28 subquestions (see online supplementary table 1). Questions encompassed three themes: (1) predatory journal definition; (2) educational outreach and policy initiatives on predatory publishing; and (3) developing technological solutions to stop submissions to predatory journals and other low-quality journals.

Questions for the first theme were informed by work identifying salient features of predatory journals ${ }^{2}$ as well as a scoping review of the characteristics of probable predatory journals. ${ }^{9}$ Questions for the remaining two themes were developed iteratively by the members of the research team. The survey was reviewed by one individual external to the research team and then pilot-tested by four others, including the one individual who reviewed the survey. Feedback received during review and piloting was incorporated into the survey.

\section{Modified Delphi process}

We used online SurveyMonkey software (http://surveymonkey.com) to deliver rounds 1 and 2 of our Delphi survey electronically. Participants were invited via an email which included key information about the study, its purpose, and how it would inform a consensus definition of predatory journals and directions for future research. Rounds 1 and 2 were available online for 3 weeks each. Two reminders were sent to participants on days 7 and 14 . Round 3 was conducted at our Predatory Summit, using the Poll Everywhere software (http://www.polleverywhere.com), where participants could respond to survey questions through live polling, watch results and participate in a face-to-face discussion.

For each of the questions, participants were asked to respond on a 9-point Likert scale (1: strongly disagree; 9: strongly agree). We chose $80 \%$ agreement as the cutoff for consensus based on findings from a systematic review of Delphi studies. ${ }^{13}$ We considered consensus to be reached if $80 \%$ of respondents scored the question within the top third (score 7-9 to include) or bottom third (score 1-3 to exclude) of the 9-point scale.

\section{Round 1}

Participants ranked the importance of all questions via the online survey. We asked participants for any additional comments they wished to provide on each question using free-text boxes.

\section{Round 2}

Based on the results and comments from round 1, the research team removed questions that reached consensus, eliminated or modified ambiguous questions, and included additional questions driven by comments from participants. For example, we received suggestions from several participants proposing that we adjust the question on collaborator roles and their ranked importance in helping to solve the problem of predatory journals. As a result, we added two additional collaborator roles that could be ranked: researchers and academic societies. We then invited participants to complete round 2 of the Delphi. In the round 2 survey invitation, we provided participants with summarised, de-identified results from round 1: a narrative summary of the survey results along with measures of central tendency (weighted average) and dispersion (range) summarised for each question. One participant requested the original comments from round 1 , which we then provided. We asked participants to again rate the importance of the remaining survey questions, using the same scale as in round 1 and referring to the results provided from round 1. Text boxes were again used to solicit additional comments.

\section{Round 3}

Participants were invited to attend our Predatory Summit to complete round 3 of the Delphi. Results from the first two rounds were available to attendees prior to the event (19-20 April 2019 in Ottawa, Canada) on the 
Open Science Framework (https://osf.io/46hwb/). We encouraged attendees to look over the summarised results, which included measures of central tendency (weighted average), dispersion (range) and comments provided by participants for each question. A final round of voting was held in person at the Summit for questions that had not reached consensus using Poll Everywhere (https://www.polleverywhere.com/). Participants could observe results in real time as data were collected. For this round, we used a 3-point Likert scale that included the same nine original responses in a simplified format (1=1-3: strongly disagree; $2=4-6$ : neutral; $3=7-9$ : strongly agree). Face-to-face presentations and discussions took place at the Summit to further refine, contextualise and finalise the results (see Summit agenda at https://osf. io/thsgw/).

\section{Participants}

Authors (group 1)

A previous scoping review identified 344 articles that discussed predatory journals. ${ }^{9}$ From these articles, we identified the corresponding authors, removed any duplicates, removed those that were members of group 2 (described in the next section), extracted author contact information, removed any authors whose contact information was not available, and sent an invitation to the remaining 198 authors to complete round 1 of our survey. Of the 198 invited authors, 72 completed the survey.

\section{Summit invitees and participants (group 2)}

Through snowball and purposive sampling of targeted experts, we identified 45 noted experts in predatory journals and journalology to participate in the Delphi process and to attend our Predatory Summit. Invitees and participants were international experts representing the varied stakeholders affected by predatory journals, including funders, academic institutions, librarians and information scientists, digital scientists, researchers involved in studying predatory journals and legitimate journals, and patient partners. Two individuals had planned to attend the Summit and so participated in rounds 1 and 2 of the Delphi, but did not attend the Summit and so could not participate in round 3.

\section{Patient and public involvement}

Since patients are the ultimate consumers of biomedical information, we felt it was critical to incorporate their opinions into this consensus process. Two patient partners were identified through their participation as partners in other (unrelated) research projects. Prior to the Delphi, they were given one-on-one educational sessions with the investigative team leads, as well as supplemental reading material. The patient partners participated in all three rounds of the Delphi. All survey responses were counted as equal contributions in the Delphi process. Results of the Delphi will be disseminated to the patient partners, who will then disseminate to their networks.

\section{Statistical analysis}

We reported discrete variables as counts/proportions. Continuous variables were reported as medians and ranges.

\section{RESULTS}

\section{Deviations from our protocol}

We did not deviate from the study procedures outlined in our protocol.

\section{Comparing round 1 results between groups 1 and 2}

The round 1 Delphi results of group 1 (authors) and group 2 (Summit invitees and attendees) were similar, with agreement on consensus or no consensus on 30 out of 35 questions. The five remaining questions reached consensus on inclusion for the Summit invitees and participants (group 2) but not for authors (group 1) (online supplementary table 2). Descriptions of the discrepancies between groups on these five items are also briefly detailed in the results below (see detailed results from round 1, group 1 at https://osf.io/vmura/; and for round 1, group 2 at https://osf.io/sry9w/; see https:// osf.io/d5463/ for a complete comparison between the results of both groups, highlighting which questions had responses that differed by more than $10 \%$ between the groups).

For reasons of feasibility and because of the similar results between the groups, as indicated in the study protocol, we invited only the Summit invitees and participants (group 2) to continue with rounds 2 and 3. We report the results of only the Summit invitees and participants (group 2) as respondents going forward.

\section{Respondent demographics}

Of the 45 Summit invitees and participants, 21 identified as female $(47 \%$; table 1$)$. There was international representation, including participants from lower-middle-income economies (India: $\mathrm{n}=1,2 \%$ ) and upper-middle-income economies (South Africa: $n=4,9 \%$ ). Summit invitees and participants reported representing a variety of stakeholder groups, with some individuals representing more than one group, including researchers $(n=22,49 \%)$, funders $(n=13,29 \%)$, policy makers $(n=2,4 \%)$, journal editors $(n=5,11 \%)$ and patient partners $(n=2,4 \%)$.

\section{Participation by round}

Of the 45 survey invitation emails sent for round 1 of the Delphi, 35 invitees completed the survey $(83 \%)$. In round 2,32 completed the survey $(76 \%)$. In both rounds, participants included detailed comments in the free-text boxes, supporting their responses or describing additional considerations on the topic, for each of the questions. Of the 43 participants who met face-to-face at the Predatory Summit, we received responses from 30 to 38 participants for each question $(70 \%-88 \%)$. The variance in response rates at the Summit could have been due to participants stepping out of the room during a question, arriving late 


\begin{tabular}{|c|c|}
\hline Characteristics & n (\%) \\
\hline \multicolumn{2}{|l|}{ Gender } \\
\hline Female & $21(47)$ \\
\hline Male & $24(53)$ \\
\hline \multicolumn{2}{|l|}{ Stakeholder group* } \\
\hline Academic institution & $4(9)$ \\
\hline Funder & $13(29)$ \\
\hline Government & $1(2)$ \\
\hline Journal editor & $5(11)$ \\
\hline Patient partner & $2(4)$ \\
\hline Policy maker & $2(4)$ \\
\hline Publisher & $3(7)$ \\
\hline Research network & $2(4)$ \\
\hline Researcher & $22(49)$ \\
\hline Student & $1(2)$ \\
\hline Other & $1(2)$ \\
\hline \multicolumn{2}{|l|}{ Geographical location } \\
\hline Canada & $24(53)$ \\
\hline India & $1(2)$ \\
\hline Italy & $3(7)$ \\
\hline The Netherlands & $1(2)$ \\
\hline South Africa & $4(9)$ \\
\hline Sweden & $1(2)$ \\
\hline Switzerland & $4(9)$ \\
\hline UK & $3(7)$ \\
\hline USA & $2(4)$ \\
\hline International & $2(4)$ \\
\hline
\end{tabular}

*Percentages do not add up to 100 since some participants identified as part of more than one stakeholder group.

or preferring not to comment on all items. A summary of all items reaching consensus, and the round at which consensus was reached, can be found in table 2 .

We review the Delphi results for each question, within each of the three categories of questions (see online supplementary table 1 for complete results).

\section{Definition of predatory journals}

Importance of developing a consensus definition for predatory journals

Consensus was reached in round 1 on the need to develop a consensus definition of predatory journals $(n=32,94 \%)$.

\section{Should the term 'predatory' be changed?}

There was no consensus on whether the term 'predatory' should be changed. Respondents were almost equally split across all lateral thirds of the Likert scale (no name change: $n=10,29 \%$; neutral: $n=13,37 \%$; alternative name required: $\mathrm{n}=12,34 \%)$. Round 2 results were similarly divided across the scale. In round 3, after inperson discussion, consensus was not reached during live voting.

\section{What alternative name(s) would you suggest?}

Consensus on an alternative name was not reached in either of the first two rounds from among the following terms: dark journals/publishers; deceptive journals/ publishers; illegitimate journals/publishers; or journals/ publishers operating in bad faith. In rounds 1 and 2, many respondents agreed that dark journals/publishers was a 'terrible name' ( $n=21,63 \%$; $n=20,67 \%)$. The name with the greatest positive traction in both rounds was deceptive journal/publisher $(\mathrm{n}=25,71 \%$; $\mathrm{n}=20,67 \%$ thought this was an 'excellent name').

After not reaching consensus in round 3 on the question of a name change, participants discussed the merits and challenges of this task. Some reasons in support of a name change included the association of predatory with the idea that the author is always a victim of a predatory journal/publisher. However, some authors publish in predatory journals knowing that the journal is predatory, for ease of publication. ${ }^{14}$ Other reasons to not use the term predatory, as was discussed at the Summit, include its affiliation with the Beall's list and the fact that other terms may be more descriptive, such as the term 'deceptive'.

Participants discussed the challenges associated with changing an established term, including challenges in identifying literature, disseminating and promoting the new name internationally, and updating existing educational materials and funder statements.

At the Summit, it was concluded that changing an already established term would likely be confusing to the scientific community and not in the best interest of moving this agenda forward. It was recommended that the term 'predatory' continue to be used and that limitations to the term, as indicated above, be recognised..$^{10}$

Characteristics that differentiate between predatory and legitimate journals

Respondents were asked to rate the importance of four different characteristics in identifying the journal as predatory. We defined characteristics as distinct features of all predatory journals. These characteristics are unique to predatory journals and generally do not occur in legitimate, high-quality OA journals. Consensus was reached for all four of the following: (1) the journal's operations are deceptive; (2) the journal's operations are not in keeping with best publication practices (eg, no membership in the Committee on Publication Ethics (COPE)) (for this item, results from group 1 (authors) were similar to group 2 (Summit invitees and participants); however, group 2 did not reach consensus (67\% thought this was a very important characteristic)); (3) the journal has low transparency regarding its operations; and (4) fake impact factors are promoted by the journal. 
Table 2 Delphi items to reach consensus as very important or strongly supported

\section{Delphi items}

Round when consensus was reached

n (\%)

1. How important is it to develop a consensus definition for predatory journals?

1 $31(94)$

2. Characteristics that differentiate predatory and legitimate journals

2a. The journal's operations are deceptive (ie, misleading; not truthful).

$33(94)$

$2 b$. The journal's operations are not in keeping with best publication practices (eg, no membership in COPE*).

2c. Journal has low transparency regarding its operations.

2d. Fake impact factors are promoted by the journal.

$28(80)$

3. Markers that best differentiate predatory and legitimate journals

3a. The journal has no retraction policy.

3b. The journal solicits manuscripts through aggressive or persuasive emails.

3c. The contact details of the publisher are not easily verifiable.

$128(80)$

. Empirically derived data that best differentiate predatory and legitimate journals

4a. The journal does not mention a Creative Commons licence.

$28(80)$
$33(94)$

$4 \mathrm{~b}$. The journal's home page has a 'look and feel' of being unprofessional.

4c. Editors and editorial board affiliations with the journal are not verifiable.

$4 d$. The journal is not a member of COPE.

5. Should public funders fund research about predatory publishing?

6. Several groups have developed checklists to help authors identify and avoid predatory publishers. Should a single, coherent checklist be developed to replace existing checklists?

7. Is there merit in developing resources or educational materials regarding predatory 3 journals/publishers in languages other than English?

\begin{tabular}{|c|c|c|}
\hline $\begin{array}{l}\text { 8. Should efforts be made to differentiate predatory journals from very low-quality } \\
\text { journals? }\end{array}$ & 1 & $30(86)$ \\
\hline $\begin{array}{l}\text { 9. Is there merit in developing a 'one stop shop' website to consolidate information, } \\
\text { training and educational materials about predatory journals? }\end{array}$ & 1 & $28(80)$ \\
\hline 10. Is there merit in establishing a predatory journal research observatory? & 2 & $24(80)$ \\
\hline
\end{tabular}

${ }^{*}$ Committee on Publication Ethics

Markers or distinguishing features that differentiate between predatory and legitimate journals

Respondents were asked to rate the importance of seven different markers in identifying a journal as predatory. We defined markers as features that are common among predatory journals. Not all markers are present in all predatory journals. Markers may be considered 'red flags' of poor journal quality. There was consensus in that three of the seven markers were very important in identifying predatory journals: (1) the journal solicits manuscripts through aggressive or persuasive emails; (2) contact details of the publisher are not easily verifiable; and (3) not having a retraction policy - this question was missed in round 2 in error, and in round 1 it almost reached consensus, with $79 \%$ of respondents rating this as a very important marker. The first two markers reached consensus in round 1, and the third marker reached consensus in round 3. The remaining four questions did not reach consensus: (4) the journal promises a very quick peer review and turnaround; (5) the journal promises rapid publication; ; (6) the journal is not a member of COPE; and (7) the journal is not listed in the Directory of Open Access Journals (DOAJ).

Empirically derived data that differentiate between predatory and legitimate journals

Respondents were asked to rate the importance of six types of empirically derived data in identifying the journal as predatory. We defined 'empirically derived' data as data resulting from experiments or statistical analyses that indicate differences between predatory journals and legitimate $\mathrm{OA}$ journals/publishers. ${ }^{2}$ In round 1, consensus was reached on four of the six questions, indicating very important data elements in identifying a predatory journal: (1) the journal's home page has a 'look and feel' of being unprofessional; (2) editors and editorial board affiliations with the journal are not verifiable; (3) the journal is not a member of 
COPE; and (4) the journal does not mention a Creative Commons (CC) licence. For this last item (journal does not mention a CC licence), results from group 1 (authors: $43 \%$ thought this was a very important characteristic) differed from group 2 (Summit invitees and participants: $80 \%$ (consensus reached) thought this was a very important characteristic). This discrepancy could be due to the fact that Summit participants, including three journal publishers and five journal editors, would be more knowledgeable about the nuances of a CC licence). The remaining two questions did not reach consensus in rounds 2 or 3: (5) the journal's APC is considerably lower than legitimate OA journals; and (6) the journal is not listed in the DOAJ.

\section{Educational outreach and policy initiatives on predatory publishing}

Should public funders fund research about predatory publishing?

In round 1, consensus was reached that public funding is essential to study and address the issue of predatory publishing $(\mathrm{n}=28,80 \%)$. Although the group of authors (group 1) did not reach consensus on this item, their responses suggest a response similar to the Summit invitees and participants ( $72 \%$ of authors thought that funding is essential).

Should research published in predatory journals be included in systematic reviews and meta-analyses?

In round 1, consensus was not reached on whether research published in predatory journals should be included in systematic reviews or meta-analyses. The research group decided to remove this question from the survey after considering the fact that respondents are not experts in systematic review or meta-analysis methodology, and therefore would not be well positioned to evaluate this item.

Do multiple checklists available for assessing predatory journals confuse prospective authors?

Consensus was not reached in any of the three rounds to determine if this was or was not a serious problem.

Should a single, coherent checklist be developed to replace existing checklists?

There was consensus in round 2 that a single checklist should be developed $(n=25,83 \%)$.

Importance of referencing and promoting pay-to-access lists indicating good-quality journals and other lists indicating potential predatory journals

Questions on good-quality lists and lists of potential predatory journals did not reach consensus in any of the three Delphi rounds. In rounds 1 and 2, half of the participants $(\mathrm{n}=17,50 \% ; \mathrm{n}=17,50 \%)$ thought it was very important to reference and promote both types of lists. In round 3, there was a switch, and more participants thought that referencing and promoting lists of potential predatory journals were more important $(n=21,58 \%)$ than referencing and promoting pay-to-access lists of good-quality journals $(n=7,23 \%)$. The change in voting could have been due to discussions at the Summit regarding pay-toaccess lists as counter to the principles of open access and equity. These discussions could have been influenced as well by the presentation by Michaela Strinzel and Anna Severin (both from the Swiss National Science Foundation), delivered at the Summit, demonstrating the overlap between lists of good-quality journals and lists of potential predatory journals. ${ }^{15}$

Ranking the level of importance of collaborators in helping solve the problem of predatory journals

In round 1, six collaborators were named and participants ranked them in order of importance: (1) academic institutions, (2) funders, (3) libraries, (4) COPE, (5) journals/publishers and (6) DOAJ. In this round, participants commented on other potential collaborators, many of whom suggested researchers and academic societies. These two categories of collaborators were added in round 2. The ranking changed slightly in this round, with the new additions, as follows: (1) academic institutions, (2) researchers, (3) journals/publishers, (4) funders, (5) libraries, (6) academic societies, for example, learned societies, (7) COPE and (8) DOAJ. Since this question did not require consensus, it was not repeated in round 3.

Merit in developing resources or educational materials regarding predatory journal/publishers in languages other than English This question almost reached consensus as an excellent idea in the first two rounds $(n=27,77 \% ; n=23,77 \%)$. The question then reached consensus in round $3(n=26,87 \%)$. Participants across the first two rounds suggested translation to other languages, including French, Spanish, Indian languages (Hindi, Bengali), German, Chinese (Mandarin) and Arabic, among others.

Strategies that would be best suited to solve the challenge of predatory journals faced by researchers in low-income and middle-income countries

Currently, the World Bank uses new classifications: lowincome, lower-middle-income, upper-middle-income and high-income economies.

Participants were asked to check options that they felt were suitable strategies. Two strategies received high response rates in round 1: a checklist to help detect predatory journals $(\mathrm{n}=26,72 \%)$; and a 'One stop shop' website that consolidates information, training and education about predatory journals/publishers $(n=30$, $83 \%)$. An error in one of the strategies listed may have contributed to false results in both rounds 1 and 2. That strategy option should have read 'Paywalled whitelists that name trustworthy or legitimate journals'; however, it read 'Paywalled whitelists that name predatory journals/ publishers'. There could have been confusion about this strategy option since whitelists in this context typically include legitimate or trustworthy journals, and not potential predatory journals or ones to avoid. In rounds 1 and 2, the journal authenticator (A usable (eg, responsive) 
browser plug-in for commonly used browsers and a backend server conceptually similar to electronic 'doughnuts' already in existence (eg, Altmetrics). The data used in the journal authenticator doughnut will be based on publication standards (eg, member of COPE) and can be used to authenticate a journal's quality status.) received high response rates as well $(n=21,58 \% ; n=23,77 \%)$. Comments from participants in the two rounds included other suggested strategies, for example, moving away from a 'publish or perish' culture in academia, which addresses the demand side of predatory journals rather than the supply side; and more support for ambassadors (eg, at the DOAJ) and the International Network for the Availability of Scientific Publications workshops onsite. A number of others indicated that they are not experts in the needs of communities in low-income and middleincome countries. Consensus was not relevant for this question and it therefore was not included in round 3.

\section{Should efforts be made to differentiate predatory journals from very low-quality journals?}

There was consensus in round 1 that important efforts should be made to differentiate between predatory journals and journals of very low quality $(n=30,86 \%)$. Although the group of authors (group 1) did not reach consensus on this item, their responses suggest a response similar to the Summit invitees and participants $(77 \%$ of authors thought that important efforts should be made). By very lowquality we mean journals that are under-resourced, or are run by an editorial board that is uninformed. These journals would not be considered predatory; however, their practices are still well below accepted publication science standards.

\section{Developing technological solutions to stop submissions to predatory journals and other low-quality journals}

Is there merit in developing a 'one stop shop' website to consolidate information, training and educational materials about predatory journals?

Consensus was reached in round 1 that a 'one stop shop' was an excellent idea $(n=28,80 \%)$. Although the group of authors (group 1) did not reach consensus on this item, their responses suggest a response similar to the Summit invitees and participants ( $76 \%$ of authors thought that developing a 'one stop shop' is an excellent idea).

\section{Is there merit in developing a journal authenticator?}

There was support in all three rounds for the development of a journal authenticator $(n=26,74 \%$; $=23,77 \%$; $=27$, $79 \%$ ); however, this question did not reach consensus.

\section{Is there merit in establishing a predatory journal research} observatory?

A data rich resource to identify ongoing research and analysis about predatory journals/publishers.

Consensus was reached in round 2 that there is strong support in establishing a predatory journal research observatory $(n=24,80 \%)$.

\section{DISCUSSION}

We conducted a modified Delphi with the aim of generating a consensus definition of predatory journals, as well as consensus on how the research community should respond to predatory journals. We came to consensus on 18 survey items out of a total of 33 (not including the question on inclusion of data in systematic reviews removed after round 1) (see table 2). These consensus items included the characteristics, markers and empirically derived data to be included in the definition of predatory journals and publishers.

In-person deliberations at the Summit proved to be an important step in coming to consensus on the decision not to change the term 'predatory'. Lengthy discussions among Summit participants centred on establishing a term that best described the activities of predatory journals and publishers, while weighing the challenges of a change in an established term. The group concluded that any change in terminology would hinder the efforts of the scholarly community to stop publication in predatory journals, and recommended continuing to use the term 'predatory'.

We were able to reach consensus on avenues of educational outreach and policy initiatives, agreeing that public funds should be allocated to research about predatory publishing, and that a single checklist should be developed to help authors detect predatory journals (see systematic review of checklists to detect predatory journals ${ }^{16}$ ). Resources such as these should be developed in languages other than English. Some agreed-on strategies to address the problem of predatory journals and publishers in low-income and lower-middle-income economies include a checklist to detect predatory journals, a 'one stop shop' website and a journal authenticator. We agreed that various collaborators have important roles in moving this agenda forward, including those identified as most responsible: academic institutions, researchers, and journals and publishers. Finally, we reached consensus that important efforts were necessary to distinguish very low-quality journals from predatory journals.

Future directions suggested included the development of technological solutions to stop submissions to predatory journals and other low-quality journals. We reached consensus on developing a 'one stop shop' website to consolidate information, training and educational materials about predatory journals and establishing a predatory journal research observatory.

The Delphi results have since been used to inform the development of a consensus statement on predatory journals and to map the next steps in addressing predatory journals. ${ }^{10}$ With this consensus definition and a roadmap for future action, we are now better positioned to study the phenomenon of predatory journals/publishers, more precisely inform policy and education initiatives, and direct resources appropriately. 


\section{Limitations}

The findings of this modified Delphi study are limited by the fact that only selected participants contributed to the survey results. Inclusion of a larger number of individuals with different expertise and backgrounds may have changed the results. We attempted to be comprehensive in the development of the survey questions; however, in compiling the final list, some questions may have been overlooked. A final limitation that may have changed the survey outcomes are possible issues with language not being preserved within the original scoping review from which we developed survey questions, or nuances in language not being captured in questions.

\section{CONCLUSION}

Bringing together international participants representing diverse stakeholder groups allowed for a comprehensive synthesis of survey responses to inform the development of a definition of predatory journals and publishers. The Delphi identified characteristics of predatory journals and publishers, education outreach and policy initiatives, as well as guidance on future directions and the development of technological solutions to stop submissions to predatory journals and other low-quality journals.

\section{Twitter Manoj Lalu@manojlalu and David Moher @dmoher}

Acknowledgements We would like to thank the following individuals at the Ottawa Hospital Research Institute for their help in reviewing and piloting the Delphi survey: Andrew Beck, Matthew McInnes, Danielle Rice and Beverly Shea. We would like to thank the following individuals who participated in the Delphi survey: Kristiann Allen, Katherine Akers, Clare Ardern, Tiago Barros, Monica Berger, Pravin Bolshete, Alison Bourgon, Marion Broome, MP Cariappa, Imti Choonara, Mary M Christopher, Marco Cosentino, Tammy Clifford, Lucia Cugusi, Lenche Danevska, Sergio Della Salla, Mahash Devnani, Helen J Dobson, Michael Donaldson, Stefan Eriksson, Matthias Egger, Kevin Fitzgibbons, Danielle Gerberi, Vinicius Giglo, Seethapathy Gopalakrishnan Saroja, Ian D Graham, Martin Grancay, Farokh Habibzadeh, Evelym Hermes-Desantis, Fang Hua, Matt Hodgkinson, Shobhit Jain, Mehrdad Jalalian, Hamid R Jamali, Manthan Janodia, Scott Kahan, Nolusindiso (Sindi) Kayi, Shawn Kennedy, SC Lakhotia, Mahlubi (Chief) Mabizela, Andrea Manca, Alexandre Martin, Ana Marusic, Stuart McKelvie, Aamir Memon, Eric Mercier, Katrin Milzow, Johann Mouton, Marilyn Oermann, Tom Olyhoek, Alexander Ommaya, Ayokunle Omobowale, Cetin Onder, Sanjay Pai, Kishor Patwardhan, Geraldine S Pearson, Alexandru Petrisor, Durga Prasanna Misra, Filipe Prazeres, Laurie Proulx, Regina Reynolds, Jason Roberts, Marc Rodger, Kem Rogers, Anna Severin, Michaela Strinzel, Mauro Sylos-Labini, Marthie van Niekerk, Jelte M Wicherts, Roger Wilson, Tim Wilson, Riah Wiratningsih and Susan Zimmerman.

Contributors CRediT (Contributor Roles Taxonomy): Conceptualisation: KDC, ML, DM. Data curation: SC. Funding acquisition: ML, KDC, DM. Methodology: KDC, ML, AG, DM. Project administration: ML, GLB, DM. Supervision: DM. Validation: ML, GLB, AG, KDC, DM. Visualisation: SC. Investigation (data collection): SC. Writing (original draft): SC. Writing (review and editing): all authors.

Funding The Predatory Summit received funding from the President's Fund, Canadian Institutes of Health Research (CIHR); Institute of Health Services and Policy Research, CIHR; Institute of Musculoskeletal Health and Arthritis, CIHR; Natural Sciences and Engineering Research Council of Canada (NSERC); Swiss National Science Foundation (grant no 174281); Social Sciences and Humanities
Research Council of Canada (SSHRC); and Office of the Vice President of Research, University of Ottawa. DM is supported by a University Research Chair (University of Ottawa). ML is supported by The Ottawa Hospital Anesthesia Alternate Funds Association

Competing interests None declared.

Patient consent for publication Not required.

Ethics approval The Ottawa Hospital Research Ethics Board (Ottawa, Ontario, Canada, 20180927-01H) (https://osf.io/ysw3g/) approved the protocol of this study.

Provenance and peer review Not commissioned; externally peer reviewed.

Data availability statement Data are available in a public, open access repository: https://0sf.io/46hwb/.

Open access This is an open access article distributed in accordance with the Creative Commons Attribution Non Commercial (CC BY-NC 4.0) license, which permits others to distribute, remix, adapt, build upon this work non-commercially, and license their derivative works on different terms, provided the original work is properly cited, appropriate credit is given, any changes made indicated, and the use is non-commercial. See: http://creativecommons.org/licenses/by-nc/4.0/.

\section{ORCID iDs}

Samantha Cukier http://orcid.org/0000-0002-4731-5662

Manoj Lalu http://orcid.org/0000-0002-0322-382X

David Moher http://orcid.org/0000-0003-2434-4206

\section{REFERENCES}

1 Shen C, Björk B-C. 'Predatory' open access: a longitudinal study of article volumes and market characteristics. BMC Med 2015:13:230.

2 Shamseer L, Moher D, Maduekwe O, et al. Potential predatory and legitimate biomedical journals: can you tell the difference? A crosssectional comparison. BMC Med 2017;15:28.

3 Manca A, Moher D, Cugusi L, et al. How predatory journals leak into PubMed. CMAJ 2018;190:E1042-5.

4 Lalu MM, Shamseer L, Cobey KD, et al. How stakeholders can respond to the rise of predatory journals. Nat Hum Behav 2017:1:852-5.

5 Moher D, Shamseer L, Cobey KD, et al. Stop this waste of people, animals and money. Nature 2017;549:23-5.

6 Priyadarshini S. India targets universities in predatory-journal crackdown. Nature 2018;560:537-8.

7 Richtig G, Berger M, Lange-Asschenfeldt B, et al. Problems and challenges of predatory journals. J Eur Acad Dermatol Venereol 2018;32:1441-9.

8 Sorokowski P, Kulczycki E, Sorokowska A, et al. Predatory journals recruit fake editor. Nature 2017;543:481-3.

9 Cobey KD, Lalu MM, Skidmore B, et al. What is a predatory journal? A scoping review [version 2; peer review: 3 approved]. F1000Res 2018;7:1001.

10 Grudniewicz A, Moher D, Cobey KD, et al. Predatory journals: no definition, no defence. Nature 2019;576:210-2.

11 Hsu C-C, Sandford BA. The Delphi technique: making sense of consensus. Pract Assess Res Eval 2007;12:8.

12 Okoli C, Pawlowski SD. The Delphi method as a research tool: an example, design considerations and applications. Inf Manage 2004:42:15-29.

13 Diamond IR, Grant RC, Feldman BM, et al. Defining consensus: a systematic review recommends methodologic criteria for reporting of Delphi studies. J Clin Epidemiol 2014;67:401-9.

14 Cobey KD, Grudniewicz A, Lalu MM, et al. Knowledge and motivations of researchers publishing in presumed predatory journals: a survey. BMJ Open 2019;9:e026516.

15 Strinzel M, Severin A, Milzow K, et al. Blacklists and whitelists to tackle predatory publishing: a cross-sectional comparison and thematic analysis. mBio 2019;10:e00411-9.

16 Cukier Set al. Checklists to detect potential predatory biomedical journals: a systematic review. medRxiv [Preprint] 2019;19010850. 